\section{International Child Health Group}

\section{ADOPTION AND IMPLEMENTATION OF TRAINING IN NEONATAL LIFE SUPPORT THROUGH SIMULATION}

${ }^{1}$ Mala Raman, ${ }^{2}$ Rupert Jones, ${ }^{3}$ Claudia Turner, ${ }^{2}$ Debra Westlake. ${ }^{1}$ University Hospitals Plymouth; ${ }^{2}$ University of Plymouth; ${ }^{3}$ Angkor Hospital for Children

\subsection{6/archdischild-2021-rcpch.674}

Background Cambodia has poor health care penetration in remote areas. The neonatal mortality rate for Cambodia is about six times higher than the UK. Many of the 3 million babies who die each year can be saved with low-tech, lowcost care. Although newborn life support (NLS) training is provided nationally, it lacks continuous practical training.

Objectives Aims

Co-development of a feasible, culturally appropriate, and acceptable newborn care simulation programme in partnership with Angkor Hospital for Children (AHC) and local health care workers in North West Cambodia.

Objectives

1. To identify gaps in knowledge, perceptions, and attitudes of healthcare workers in providing evidence-based NLS.

2. To address barriers and facilitators, including human factors such as team working, communication and newborn care practice routines in the local cultural setting.

3. To co-develop, deliver, and evaluate a digital NLS training package using mannequins.

Methods Stage 1 - Research

Qualitative data was collected through observation of newborn care in two health centres, focus groups $(\mathrm{n}=\mathrm{x}$ participants) and semi-structured interviews $(n=4)$. Rapid appraisal techniques were applied. Data was iteratively collected and analysed in the field by a team of international and local clinical researchers in order to understand local contextual factors. Framework analysis was used by the team to organise and analyse the data generated. Co-production ensured we were cognisant of factors that could influence the adoption of the training and guide development of training methods for health care workers.

Results Health care workers understood the need for regular practice. However, barriers included a shortage of functioning equipment, layout of the labour room, lack of guidelines for equipment checks and routine practice of newborn resuscitation. Factors impeding scheduled practice included work routine, lack of simulation equipment, understanding of health care workers on prioritisation of their role and the local staffing.

Gaps in knowledge were in the areas of recognising the signs of life, understanding the causes of newborn death and cardiac compression as an essential component of resuscitation.

All respondents to the study were enthusiastic about further NLS training and identified it as a key need. Team working and dynamics were identified as important facilitators of learning and practice routines.

Research findings informed co-development of a train-thetrainer programme with local partners which were delivered to workers from 16 centres.

Stage 2 - research

A programme was co-designed with local health care workers and implemented despite the pandemic. To deliver high- quality, sustainable training with inbuilt monitoring and evaluation, a bespoke blended learning system was used (www. the625/teach box). Teach Box does not require connection to electricity or Wi-Fi for delivery. Participating centres were provided with Andriod tablets, low-fidelity simulation kits and posters.

The system remains in regular use in all 16 centres. Conclusions A culturally appropriate digital low-fidelity teaching model can be implemented in a remote low resource setting. This model could have an impact on newborn care and survival in this and other settings.

\section{MEETING THE HEALTH NEEDS OF UNACCOMPANIED ASYLUM SEEKING CHILDREN: A SCOPING REVIEW OF ROUTINE HEALTH ASSESSMENT}

Behrouz Nezafat Maldonado, Sarah Al-Rawi, Bhanu Williams. London North West University Healthcare NHS Foundation Trust

\subsection{6/archdischild-2021-rcpch.675}

Background Approximately, 34 million of 79.5 million forcibly displaced persons globally are minors, many of whom are unaccompanied asylum seeking children (UASC). UASC often experience unmet health needs, physical harm or emotional trauma in their birth country, or during their journey to seek asylum. International guidelines recommend health screening for all migrant children.

Objectives

1. Scope the literature on UASC routine health assessment across Australia, European Economic Area, Canada, New Zealand, Switzerland, United Kingdom and United States $(n=36)$.

2. Analyse health screening programmes to inform future health policy.

Methods Literature published (2010-March 2021) was systematically searched across MEDLINE, Web of Science, Cochrane and CINAHL. Records were screened against the inclusion criteria. A framework analysis across national and international organizations' websites identified additional documents.

Results 33 of 1432 records were included. UASC screening was reported across 13/36 countries, summarised in table 1 . Commonly, studies were observational reports lacking references to clinical guidelines.

Tuberculosis disease screening was offered in 12/13 countries. 8/13 countries used a risk-based approach, meaning UASC exposed to risk factors or from areas endemic for conditions (such as tuberculosis, hepatitis or parasites) were screened.

Non-communicable disease screening guidance was present across 10/13 countries. $7 / 13$ proposed blood testing for anaemia and nutritional deficiencies. Dental checks and developmental assessments were suggested in $7 / 13$. Mental health screening was recommended in 6/13 countries.

Conclusions Studies reporting UASC health assessment were not found for all countries. We found considerable procedural variation, which can exacerbate health inequalities. While we do not assume UASC health needs in these countries are not addressed, it raises concerns of the standards followed. Countries reporting screening may represent settings where optimal assessment is being followed. However, no audit data are available to compare guideline implementation and effectiveness. Frequently, emphasis was placed on infectious disease screening whilst other health components like mental health were less commonly addressed. 